\title{
Curvature of micropores in Al-Cu alloys: An X-ray tomography study
}

\author{
M. Felberbaum*, M. Rappaz \\ Laboratoire de Simulation des Matériaux, Ecole Polytechnique Fédérale de Lausanne, Station 12, Lausanne CH-1015, Switzerland
}

Received 6 May 2011; received in revised form 29 June 2011; accepted 2 July 2011

Available online 2 September 2011

\begin{abstract}
Micropores formed in Al-Cu alloys cast under controlled conditions have been analyzed using high-resolution X-ray tomography. The influence of inoculation conditions, copper content, cooling rate and initial hydrogen content on the morphology of pores has been investigated. Based on the three-dimensional reconstructed shape of the pores, the distribution of curvature was estimated. It is shown that the mean curvature of pores in either non-inoculated or inoculated $\mathrm{Al}-4.5 \mathrm{wt} . \% \mathrm{Cu}$ alloys can be as large as $0.35 \mu \mathrm{m}^{-1}$ near the end of solidification and can be fairly well approximated by a set of interconnected cylinders growing in between the primary phase dendrites. The so-called "pinching" effect, i.e. the restriction of the pore curvature by the solid network, is a function of the volume fraction of the primary phase and of the secondary dendrite arm spacing. If the fraction of porosity is highly dependent on the initial hydrogen content, the curvature itself is only weakly influenced by this parameter. Based on these results, it is concluded that curvature plays a major role in porosity models and that the analytical pinching model developed by Couturier et al. [1] offers a fairly good and simple approximation of this contribution.
\end{abstract}

(C) 2011 Acta Materialia Inc. Published by Elsevier Ltd. All rights reserved.

Keywords: Microporosity; Curvature; X-ray tomography; Pinching model; Solidification

\section{Introduction}

Porosity is one of the major defects in castings; it reduces the mechanical properties of the cast species, in particular ductility and fatigue resistance, and impairs its surface quality [2]. In order to minimize the formation of such defects in castings, computational modeling is now widely used in many foundries. Although porosity is a complex problem, simple criteria such as the well-known Niyama criterion are often applied, usually on the basis of the thermal field [3]. In more advanced models, the pressure drop within the mushy zone induced by solidification shrinkage, the solubility and diffusion of dissolved gases, and the nucleation and growth of pores are accounted for. Details of these mechanisms can be found in the review of Lee et al. [4] and in the textbook by Dantzig and Rappaz [3].

\footnotetext{
* Corresponding author.

E-mail address: milan.felberbaum@a3.epfl.ch (M. Felberbaum).
}

While a lot of attention has been paid to pressure drop calculations and gas segregation, several aspects related to pore nucleation and growth remain unclear. It has been shown that nucleation of microporosity can be highly influenced by the presence of foreign impurities, such as oxide bifilms [2]. On the other hand, the growth (and thus fraction) of porosity is limited by two other aspects, namely the diffusion of dissolved gases and the curvature of the pores, which have not been studied extensively. Indeed, if the pore density is low and the solidification time is fairly short, i.e. small Fourier number for the diffusion of gases, the mushy zone can remain supersaturated, thus reducing the pore fraction as shown by Carlson et al. [5]. This effect becomes even more pronounced at high solid fractions since the average diffusion coefficient of gases in the mushy zone decreases [6]. On the other hand, a pore constrained to grow in between narrow interdendritic liquid channels has a higher curvature, and thus a higher internal pressure, than a free-growth spherical pore. As more gas can be stored in this compressible phase, the fraction of porosity hence decreases with an increasing curvature. While this 
effect was recognized long ago, for example by Carpentier [7], there are almost no basic studies to support the various geometrical models that have been made for the growth restriction (so-called "pinching") of pores by the existing solid network $[8,9,1]$.

The advent of high-resolution X-ray tomography offers tremendous possibilities for the three-dimensional (3-D) reconstruction and visualization of morphologies [10]. The in situ formation of microporosity has already been observed by X-ray radiography [11] or tomography [12], but with limited spatial resolution $(2.5$ and $15 \mu \mathrm{m}$, respectively). It should be noted that Lee et al. [13] pioneered such observations, using microfocus radiography with a resolution of $25 \mu \mathrm{m}$. Nowadays, submicron resolution can easily be achieved on most synchrotron X-ray tomography beamlines. Combined with increasingly fast data acquisition systems, these techniques allow in situ microtomography observations of solidifying microstructures [14], even under tensile deformation [15].

In order to better understand and evaluate the contribution of pore curvature, the present study is focused on the $\mathrm{X}$-ray tomography characterization of the 3-D morphology of micropores in $\mathrm{Al}-\mathrm{Cu}$ alloys after complete solidification under various controlled conditions. Compared to in situ observations, such observations have the drawback that the pore evolution is unknown and only give access to the final shape. But this has also two advantages. First, the resolution can be optimum (typically $0.74 \mu \mathrm{m}$ pixel $^{-1}$ in the present case), thus allowing a more precise 3-D reconstruction which can then be used to evaluate accurately the curvature distribution of the pores. Second, the solidification conditions can be those of actual castings, and thus the influence of parameters such as inoculation conditions, copper content, cooling rate and initial gas content can be investigated. As will be shown, the measured curvature distribution of the pores will be put into relation with the microstructure characteristics and with some of the available analytical pinching models.

\section{Theory}

Microporosity formation is due to two concomitant mechanisms: solidification shrinkage and gas segregation/ precipitation [16]. Both solidification shrinkage and thermal contraction induce a liquid flow towards the root of the mushy zone, producing a pressure drop in the liquid (the liquid can even be under tension, i.e. negative pressure [17]). On the other hand, hydrogen segregation occurs since the partition coefficient between the solid and liquid alloy is much smaller than 1 ( $k_{0 H}=0.07$ for $\mathrm{H}$ in pure $\left.\mathrm{Al}[18]\right)$.

Assuming very fast diffusion of hydrogen, the volume fraction of pores can be calculated using the rule [3]:

$\left\langle\rho_{0}\right\rangle C_{H 0}=\left(g_{s} \rho_{s} k_{0 H}+\left(1-g_{s}\right) \rho_{\ell}\right) C_{H \ell}+2 \frac{g_{p} p_{g}}{\mathcal{R} T}$

where $g_{s}$ and $g_{\ell}$ are the volume fractions of solid and liquid, respectively, $\rho_{s}$ and $\rho_{\ell}$ their density, $\left\langle\rho_{0}\right\rangle$ the pore-free density of the material $\left(=g_{s} \rho_{s}+g_{\ell} \rho_{\ell}\right), C_{H 0}$ the initial hydrogen composition of the alloy, $g_{p}$ the pore volume fraction, $p_{g}$ the pressure inside the pore, $\mathcal{R}$ the constant gas law and $T$ the temperature. $C_{H \ell}$ is the composition of hydrogen in the liquid given by Sievert's law [3]:

$C_{H \ell}=\left(A_{H} 10^{2\left(e_{H}^{I} C_{\ell}+r_{H}^{I} C_{\ell}^{2}\right)}\right)^{-\frac{1}{2}} \exp \frac{\Delta H_{H}^{m}}{2 R T}\left(\frac{p_{g}}{p_{0}}\right)$

where $A_{H}$ is a constant, $C_{\ell}$ the liquid copper composition, $e_{H}^{I}$ and $r_{H}^{I}$ the first- and second-order interaction coefficients between $\mathrm{Cu}$ and $\mathrm{H}$ given by Ref. [19], $\Delta H_{H}^{m}$ the enthalpy of mixing between hydrogen and copper, and $p_{0}$ the reference pressure (see Ref. [3] for more details). Although the pressure in the liquid $p_{\ell}$ can be negative, the pressure in the pore $p_{g}$ is of course positive and the difference between the two is given by the Laplace-Young equation:

$p_{g}=p_{\ell}+2 \gamma_{\ell g} \bar{\kappa} \quad$ with $\quad \bar{\kappa}=\frac{1}{2}\left(\frac{1}{R_{1}}+\frac{1}{R_{2}}\right)$

where $\bar{\kappa}$ is the local mean curvature defined by the local principal radii of curvature $R_{1}$ and $R_{2}$ [20]. Once a pore has nucleated in the liquid, it adopts a spherical shape and $\bar{\kappa}=1 / R_{p}$. In this case, Eq. (3) becomes $p_{g}=p_{\ell}+2 \gamma_{\ell g} / R_{p}$ and the pore fraction $g_{p}$ can then be taken as:

$g_{p}=\mathcal{N}_{p} \frac{4}{3} \pi R_{p}^{3}$

where $\mathcal{N}_{p}$ is the pore density. Therefore, if $p_{\ell}$ is known (either atmospheric pressure or obtained from a coupling with fluid-flow calculations), Eqs. (1)-(4) are complete, assuming $\mathcal{N}_{p}$ is known from a nucleation model.

However, in many situations the pore has to develop in a mushy region and cannot displace the solid network. Therefore, the pore is forced to adopt a non-spherical shape which is the "negative" of the solid already formed, in which case $g_{p}$ or $\bar{\kappa}$ and $p_{g}$ are no longer related by a simple relationship. On the other hand, as shown schematically in Fig. 1, the pore pressure is unique and thus the liquid-gas interface must have a unique curvature (see Eq. (3)). ${ }^{1}$

In order to account for the pinching of a pore by the solid network, porosity models replace the simple $g_{p}-\bar{\kappa}$ relationship of Eq. (4) by a dependence $\bar{\kappa}\left(g_{\alpha}, \lambda_{2}\right)$ or $\bar{R}_{p}\left(g_{\alpha}, \lambda_{2}\right)$, where $g_{\alpha}$ is the volume fraction of the primary phase, $\lambda_{2}$ its characteristic length scale (e.g. secondary dendrite arm spacing) and $\bar{R}_{p}$ an equivalent radius of curvature associated with $\bar{\kappa}$. Fig. 2 shows various models developed for the normalized equivalent pore radius $\bar{R}_{p} / \lambda_{2}=\left(\kappa \lambda_{2}\right)^{-1}$ as a function of the primary phase fraction $g_{\alpha}[8,9,1]$. Poirier et al. [8] and Péquet et al. [9] developed models based

\footnotetext{
${ }^{1}$ The pressure in the pore $p_{g}$ is certainly unique, since pressure variations in this gas phase must be equilibrated at the speed of sound, i.e. almost instantaneously. But assuming $\bar{\kappa}$ to be uniform at the liquid-gas interface of a pore also requires having $p_{\ell}$ to be uniform around a pore, which might not be true due to much slower/viscous liquid motion.
} 


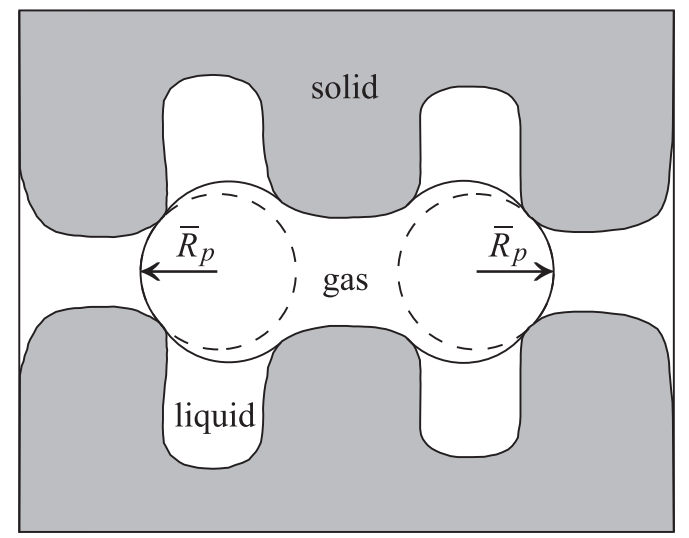

Fig. 1. 2-D schematic view of pore growth between dendrite arms, showing the principle of a uniform curvature (or radius) of the liquid-gas interface.

on the liquid space left in between a 1-D and 2-D hexagonal network of dendrites. Couturier et al. [1] considered (infinitely long) cylindrical secondary dendrite arms arranged in a square lattice, in between which a perfectly non-wetting pore can develop. The radius $\bar{R}_{p}\left(g_{\alpha}\right) / \lambda_{2}$ in this case is given by half the diagonal of the free liquid space left in between the arms (see insert). Once a spherical pore formed in between these arms reaches this maximum size, it propagates along the arms with a nearly cylindrical shape, the ends of which still have the mean curvature given by this model. Couturier et al. also developed a pinching model for spherical equiaxed grains. Finally, Fig. 2 shows with arrows the volume fraction of the primary phase $g_{\alpha}\left(T_{\text {eut }}\right)$ of the three alloys investigated in the present study, at the eutectic temperature $T_{\text {eut }}$ (see next section).

\section{Experiment}

Three alloy compositions were investigated in this study: Al-1 wt. $\% \mathrm{Cu}$ (without inoculant), $\mathrm{Al}-4.5 \mathrm{wt} . \% \mathrm{Cu}$ (with or without inoculant) and $\mathrm{Al}-10 \mathrm{wt} . \% \mathrm{Cu}$ (without inoculant). They were prepared by mixing suitable amounts of pure Al $(99.995 \%)$ and oxygen-free high-conductivity (OFHC) copper pieces, after careful deoxidation, cleaning and drying (see Ref. [21] for more technical details). These alloys were then directionally solidified (DS) using two different set-ups as described below.

The first set-up - termed $D S_{1 \mathrm{D}-\mathrm{ATM}}$ - was designed so that the oxide skin at the surface of the melt was never broken, but solidification occurred under atmospheric conditions, i.e. the hydrogen partial pressure, which is a key factor for porosity formation in Al alloys, was not controlled. This device, similar to that of Ref. [22], was used only for the casting of $\mathrm{Al}-4.5 \mathrm{wt} . \% \mathrm{Cu}$ (with and without inoculant). In the second approach - termed $D S_{1 \mathrm{D}-\mathrm{AC}}-$ the 1-D-DS set-up was placed inside a vacuum induction furnace in which the atmosphere can be controlled. However, the melt had to be poured in this case, leading to possible oxide entrapment during the casting process. However, since the atmosphere was reducing in this case,

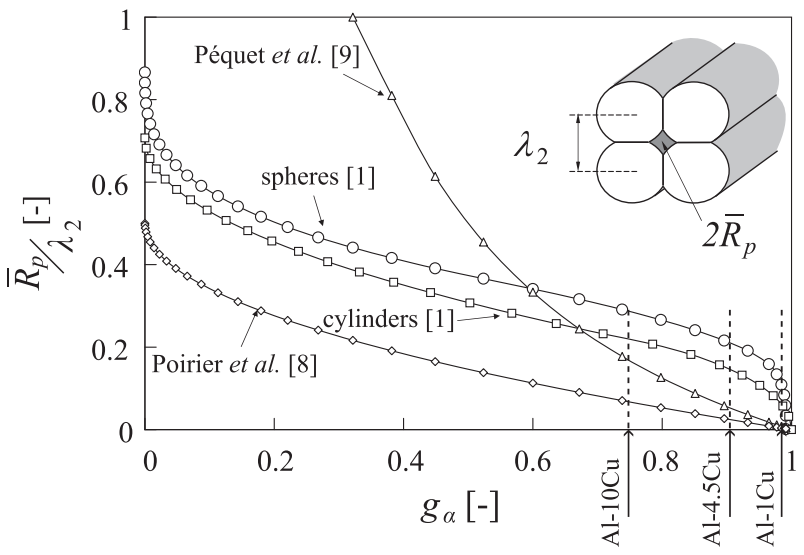

Fig. 2. Equivalent pore radius of curvature $\bar{R}_{p}$ normalized by the secondary dendrite arm spacing $\lambda_{2}$ (dendrite morphology) [1], grain diameter $R_{g}$ (globular equiaxed structure) [1], or following the developments of Refs. [8] or [9], as a function of the volume fraction of primary phase $g_{\alpha}$. The insert shows the geometry for the first case. Note also the vertical arrows representing $g_{\alpha}\left(T_{\text {eut }}\right)$ for each composition (in wt.\%) investigated in this study.

the mechanism of oxide bifilm entrainment described by Campbell is certainly minimized [2].

The $D S_{1 \mathrm{D}-\mathrm{ATM}}$ set-up comprised a cylindrical (slightly conical) $60 \mathrm{~mm}$ diameter $\times 110 \mathrm{~mm}$ height stainless steel mold coated with boron nitride $(\mathrm{BN})$ to avoid chemical reaction with the melt. At the bottom, a coated stainless steel sheet ( $2 \mathrm{~mm}$ thick) was mechanically fixed to the cylindrical mold. A cover holding several K-type thermocouples of various lengths was placed at the top of the mold. The temperatures were recorded before and during solidification using a NetDAQ Data Acquisition system (FLUKE Corporation, Everett, WA). The entire system containing the alloy was placed for about $1 \mathrm{~h}$ in a furnace set-up $50 \mathrm{~K}$ above the liquidus of the alloy. It was then taken out and clamped onto a platform that had a hole in the center. A water jet spray placed under this hole was turned on to induce nearly 1-D upward solidification, since the lateral and top surfaces of the mold were wrapped with a thick quartz wool layer. ${ }^{2}$ The ambient temperature and relative humidity were recorded during the experiment.

The $D S_{1 \mathrm{D}-\mathrm{AC}}$ set-up was used for the three alloys, without inoculation, in order to investigate the influence on the pore morphology of copper content, cooling rate and initial hydrogen content. The mold consisted of an internal ceramic cylinder $(3 \mathrm{~mm}$ thick, $40 \mathrm{~mm}$ diameter, $70 \mathrm{~mm}$ height), placed within an outer stainless steel mold. Four heating cartridges were placed in the steel mold so as to control the mold surface temperature and to reduce radial heat transfer. The ceramic cylinder was closed at the bottom by a copper chill cooled down with water. The temperatures before and during solidification were recorded by

\footnotetext{
${ }^{2}$ A temperature difference of only about $1 \mathrm{~K}$ was measured between three thermocouples placed at the same height but at different axial positions.
} 


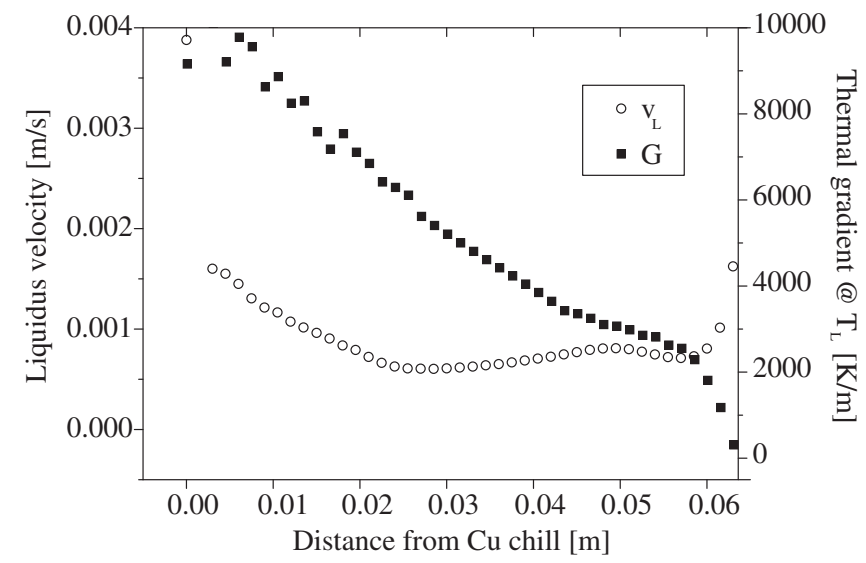

Fig. 3. Thermal gradient and liquidus velocity for an $\mathrm{Al}-4.5 \mathrm{wt} . \% \mathrm{Cu}$ casting experiment performed under $800 \mathrm{mbar}$ of $\mathrm{N}_{2}-10 \mathrm{vol} . \% \mathrm{H}_{2}$, with an initial temperature of $1073 \mathrm{~K}$, as a function of the height within the specimen.

four K-type located at different heights within the mold and connected to a NetDAQ Data Acquisition system.

The alloy contained in a quartz crucible was first melted by induction in the same vacuum chamber containing this mold. It was kept molten for $20 \mathrm{~min}$ in the presence of a pressure of $\mathrm{N}_{2}-10 \mathrm{vol} . \% \mathrm{H}_{2}$ gas. With the strong convection induced by induction, it is assumed that the melt reached equilibrium with the partial pressure of hydrogen, so that the hydrogen composition of the melt can be calculated. ${ }^{3}$ The alloy was then poured and solidified in the ceramic mold, previously preheated to the initial melt temperature.

First the influence of the alloy copper content and cooling rate on porosity was investigated. For that purpose, $\mathrm{Al}-1$ wt. $\% \mathrm{Cu}, \mathrm{Al}-4.5$ wt. $\% \mathrm{Cu}$ and $\mathrm{Al}-10$ wt. $\% \mathrm{Cu}$ samples have been solidified under the same hydrogen partial pressure ( 800 mbar of $\mathrm{N}_{2}-10 \mathrm{vol} . \% \mathrm{H}_{2}$ ) from an initial melt temperature of $1073 \mathrm{~K}$. The influence of the initial hydrogen composition on porosity was analyzed next, using a non-inoculated $\mathrm{Al}-4.5 \mathrm{wt} . \% \mathrm{Cu}$ alloy solidified under the same hydrogen partial pressure $\left(800 \mathrm{mbar}\right.$ of $\mathrm{N}_{2}$ $10 \mathrm{vol} . \% \mathrm{H}_{2}$ ), but with two different initial melt temperatures, namely 973 and $1073 \mathrm{~K}$. The corresponding calculated initial hydrogen contents were 0.23 and 0.41 $\mathrm{cc}_{\text {STP }} 100 \mathrm{~g}^{-1}$, respectively (see Eq. (2)). A reference sample without (or with minimum) hydrogen was also cast under pure Ar from an initial temperature of $1073 \mathrm{~K}$.

The thermal gradient $G$ and the liquidus velocity $v_{L}$ of the DS set-ups were determined using a 1-D inverse modeling technique [25] using the software CalcoSOFT (CalcoSOFT is a registered software produced by Calcom ESI Group, Lausanne, Switzerland). Fig. 3 shows a typical result for an $\mathrm{Al}-4.5 \mathrm{wt} . \% \mathrm{Cu}$ casting experiment made with the $D S_{1 \mathrm{D}-\mathrm{AC}}$ set-up. The thermal gradient $G$ is about $9000 \mathrm{~K} \mathrm{~m}^{-1}$ near the bottom of the mold, whereas $G \rightarrow 0$ around $z=0.07 \mathrm{~m}$. This last value shows that the top sur-

\footnotetext{
${ }^{3}$ Refs. $[23,24]$ mention 5 and $25 \mathrm{~min}$ as the gas equilibration times for Al- and Fe-base alloys, respectively.
}

face of the mold is well insulated. The velocity of the liquidus $v_{L}$ stays nearly constant at around $1 \mathrm{~mm} \mathrm{~s}^{-1}$. This thermal calibration will enable us to determine the secondary dendrite arm spacing as a function of the location within the specimen [3].

\section{X-ray tomography and data analysis}

In order to view the microstructure and micropores with X-ray tomography, cylindrical samples $(1.4 \mathrm{~mm}$ diameter, $1 \mathrm{~mm}$ height) were extracted at specific heights from the castings: $8 \mathrm{~mm}$ from the bottom chill of the $D S_{1 \mathrm{D}-\mathrm{ATM}}$ castings; 10 and $40 \mathrm{~mm}$ from the bottom chill of the $D S_{1 \mathrm{D}-\mathrm{AC}}$ castings. For further details of this analysis technique, see e.g. Refs. [26,27]. In the present case, the tomography experiments were conducted on the ID19 beamline of ESRF, Grenoble, and on the TOMCAT beamline of SLS, Villigen, using absorption contrast only. In this case, the 3-D reconstruction is based on the local absorption of a small volume element (voxel), which is a function of the local density and atomic number of the chemical elements present in this voxel [28]. A total of $1501 \mathrm{X}$-ray projections were recorded (with a pixel size of $0.74 \mu \mathrm{m}$ ) for each specimen.

After reconstruction, an X-ray tomography stack containing the information of the grey level (integer from 0 to 255) as a function of the voxel position $(x, y, z)$ is obtained. A typical 2-D cross-section of such a stack is given in Fig. 4. In this reverse-contrast figure, black regions correspond to a local absorption close to 0 , and thus represent the pore phase. White regions correspond to high absorption voxels, in this case the $\mathrm{Al}-\mathrm{Al}_{2} \mathrm{Cu}$ eutectic. Note that the fine eutectic structure is barely resolved in the enlarged view of Fig. 4b. Finally, the grey regions correspond to the Al matrix phase. Note that the thermal gradient $G$, which by convention in this work is parallel to the $z$ axis of a reference frame, is pointing towards the reader.

Looking at Fig. 4b, several isolated pores can be seen in this cross-section, but as will be seen later, they are in fact interconnected in 3-D. In order to extract the 3-D information of the pore phase, the stack of images is first cropped to a typical size of $500 \times 500 \times 500$ voxels, and then binarized so that the grey $\mathrm{Al}$ matrix and the eutectic regions are turned white, while the pore remains black. These cropping and threshold operations are standard procedures implemented in the freeware ImageJ [29]. Next, a 3-D median filter is applied to the volume in order to suppress isolated noise without blurring sharp edges. (Specifically, the median filter replaces a pixel by the median of all pixels in the neighborhood.) Various routines of image analysis were then performed on this volume that we call $V_{\text {init }}$; these are described in the following.

\subsection{Pore morphology}

In order to determine whether a pore is elongated or rather has an "equiaxed" shape, the moments of the inertia 

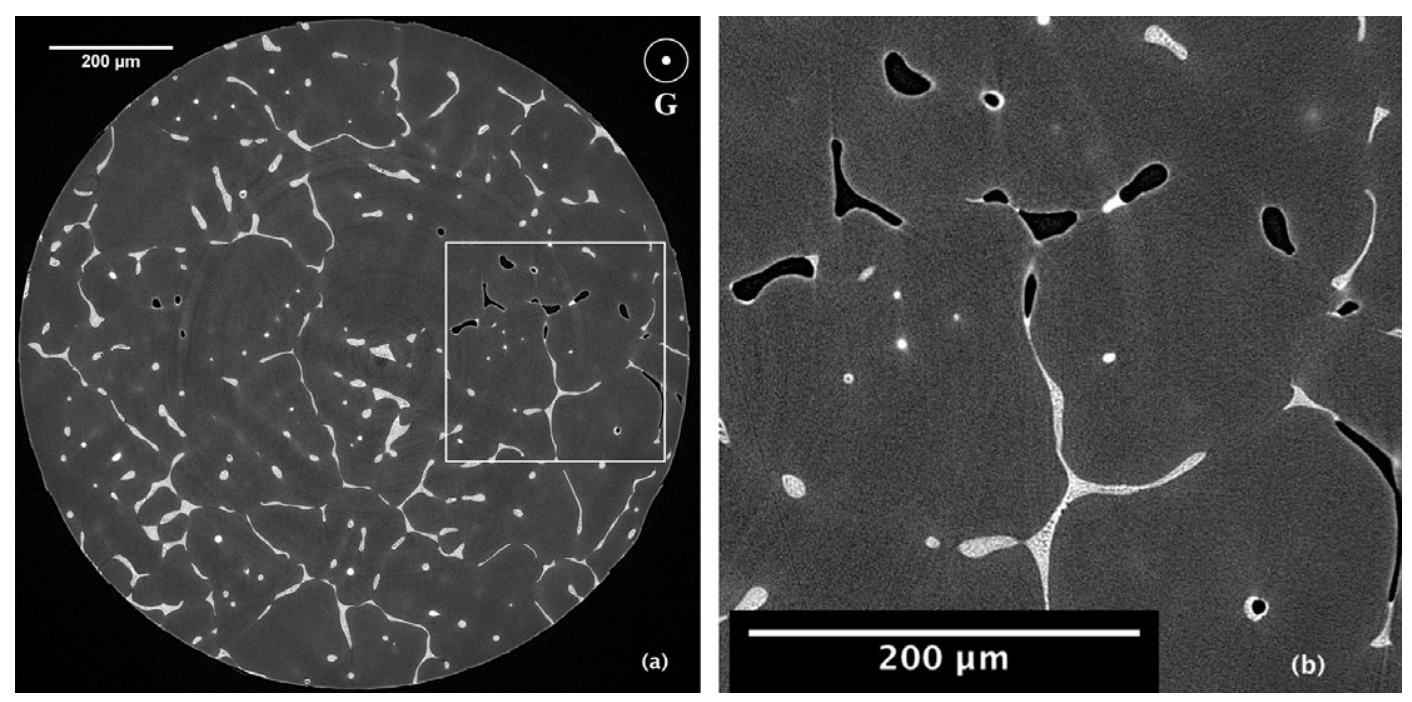

Fig. 4. (a) Cross-section of a typical $\mathrm{Al}-4.5 \mathrm{wt} . \% \mathrm{Cu}$ sample observed by X-ray tomography (after reconstruction), with (b) an enlarged region. White regions correspond to the eutectic, dark grey regions to the $\mathrm{Al}$ matrix and black regions to the pore phase. The thermal gradient $G$ points towards the reader. Note the small distinct pores in the enlarged region that are, in fact, interconnected (see Fig. 7b which shows the 3-D rendering of the same pore).

tensor matrix, together with its principal directions, have been calculated. Following a method similar to that developed by Doube et al. [30], we define the moments of the inertia tensor matrix of a pore as:

$$
I\left[\mu \mathrm{m}^{5}\right]=V_{V} \sum_{k=1}^{N_{k}}\left(\begin{array}{ccc}
y_{k}^{2}+z_{k}^{2} & -x_{k} y_{k} & -x_{k} z_{k} \\
& x_{k}^{2}+z_{k}^{2} & -y_{k} z_{k} \\
\operatorname{sym} & & x_{k}^{2}+y_{k}^{2}
\end{array}\right)
$$

where $V_{V}$ is the volume of one voxel and the summation is performed over the $N_{k}$ voxels belonging to the same pore, i.e. their grey level is 0 . The coordinates $\left(x_{k}, y_{k}, z_{k}\right)$ of each voxel $k$ are measured in a reference frame centered on the "gravity" center of the pore and with the $z$-axis parallel to $G$. This tensor has three eigenvectors with their three associated eigenvalues. Since this moments of inertia tensor is given in $\mu \mathrm{m}^{5}$, it has been non-dimensionalized to give the tensor $I^{N D}$, by normalizing by the pore volume and by an average distance to its center. This ensures $I^{N D}$ to be influenced by the pore shape only, and not by its volume. In that case, it is easy to show that $I_{z z}^{N D}$ is equal to $2 / 3$ for a spherical pore. Accordingly, the tensor $I^{N D}$ has been multiplied by $3 / 2$ in order to have 1 on the diagonal terms and 0 for the off-diagonal terms for the case of a spherical pore.

As will be seen in the next section, the principal moments of inertia depend very much on the pore morphology, columnar or equiaxed, of the $\mathrm{Al}-\mathrm{Cu}$ samples.

\subsection{Curvature calculations}

As curvatures correspond to second derivatives of a surface [31], they can hardly be measured with sufficient accuracy from discrete voxels. ${ }^{4}$ Therefore, it is first necessary to smooth the surface outlined by the "stair-like" layer made of discrete voxels missing a neighbor (called hereafter "voxsurface"). Since various smoothing procedures can produce quite different curvature results, the same standard procedure was applied on a given stack of voxels. ${ }^{5}$

We used the possibility offered by the software Avizo (Avizo is a registered trademark of Mercury Computer Systems, Chelmsford, MA) to replace the voxsurface by a triangular approximation. After application of a "constrained smoothing" filter, a module that computes the curvature for a smoothed surface made of triangles was applied. In a nutshell, the algorithm traverses all triangles of the surface. For each triangle, the algorithm finds a quadratic surface approximation based on its neighbors, and evaluates the eigenvalues/eigenvectors of the quadratic surface. Indeed, calling $w$ the normal to this surface and $u$ and $v$ the two unit vectors of the tangent plane corresponding to the principal curvature axes, the surface is locally given by:

$w(u, v)=2 \kappa_{1} u^{2}+2 \kappa_{2} v^{2}$

where $\kappa_{1}$ and $\kappa_{2}$ are the two local principal curvatures. From these two values, the mean curvature $\bar{\kappa}$ and the Gauss curvature $\mathcal{G}$ can be computed [31]:

$\bar{\kappa}=\frac{1}{2}\left(\kappa_{1}+\kappa_{2}\right) \quad$ and $\quad \mathcal{G}=\kappa_{1} \kappa_{2}$

Once $\kappa_{1}$ and $\kappa_{2}$ were calculated at each point of the pore surface, they were used to color the 3-D surface with either the mean or the Gauss curvature. They were also sorted as follows in order to plot interfacial shape distributions (ISD), as described by Kammer et al. [20]. First, as the tomography resolution was about twice the voxel size

\footnotetext{
${ }^{4} \mathrm{~A}$ box count technique is sometimes used to calculate the curvature from voxels, but the accuracy of this approach is rather poor.
} 


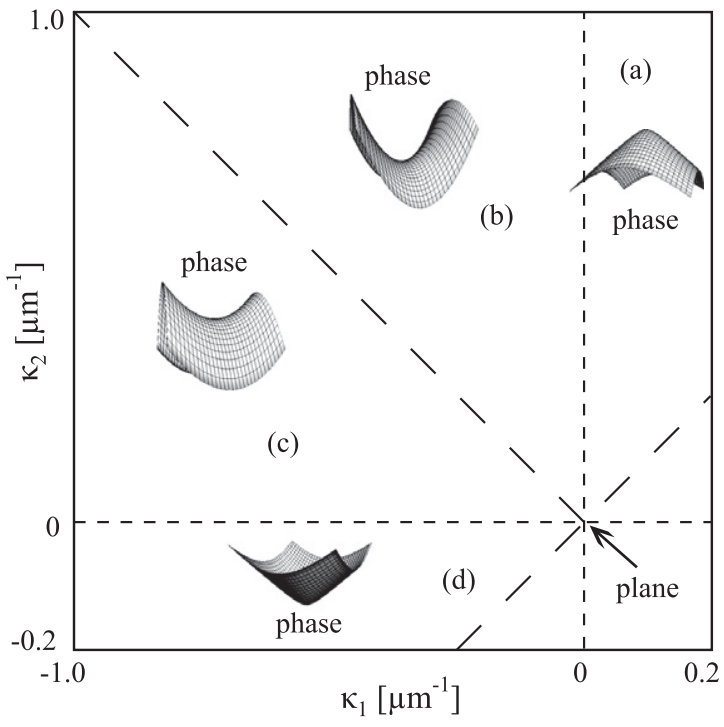

Fig. 5. $\kappa_{1}-\kappa_{2}$ graph for the representation of the interfacial shape distribution (ISD) contour plot [20].

$(0.74 \mu \mathrm{m})$, the pairs of $\left(\kappa_{1} ; \kappa_{2}\right)$ that had a local mean curvature, in absolute value, larger than $\frac{1}{2 \cdot 0.74} \mu \mathrm{m}^{-1}$ were discarded. If needed, the values $\left(\kappa_{1} ; \kappa_{2}\right)$ at each point were then permuted so that $\kappa_{2} \geqslant \kappa_{1}$. The number $N_{i j}$ of curvature couples falling within predefined classes ([ $\left[\kappa_{1 i}\right.$, $\left.\left.\kappa_{1 i}+\Delta \kappa\right],\left[\kappa_{2 j}, \kappa_{2 j}+\Delta \kappa\right]\right)$ were then counted, with a weighting factor proportional to the surface of the corresponding triangle of the triangular surface. Finally, isovalues were drawn in a $\left(\kappa_{1} ; \kappa_{2}\right)$ diagram such as the one shown in Fig. 5. In such a diagram, various local pore morphologies (convex, saddle, concave) are also shown based on both $\bar{\kappa}$ and $\mathcal{G}$. Although not represented graphically here, a locally spherical pore surface corresponds to $\kappa_{1}=\kappa_{2}$ (dashed line at $45^{\circ}$ passing through the origin), while a locally cylindrical morphology is such that $\kappa_{1}=0$ and $\kappa_{2}>0$ (vertical dashed line) for a convex pore surface, and $\kappa_{1}<0$ and $\kappa_{2}=0$ (horizontal dashed line) for a concave one. In addition, the following characteristic values were calculated and represented on each ISD plot:

(i) The average values of both principal curvatures $\left\langle\kappa_{1}\right\rangle_{t o t}$ and $\left\langle\kappa_{2}\right\rangle_{t o t}$ for the whole set of the $\left(\kappa_{1}, \kappa_{2}\right)$ pairs.

(ii) The average values of both principal curvatures $\left\langle\kappa_{1}\right\rangle_{\geqslant 0}$ and $\left\langle\kappa_{2}\right\rangle_{\geqslant 0}$ for the positive values of the $\left(\kappa_{1}, \kappa_{2}\right)$ pairs only.

(iii) The surface-to-volume ratio of each pore defined as $S_{V}=S_{p} / V_{p}$, where $S_{p}$ and $V_{p}$ are the surface and volume of the pore, respectively.

Finally, it must be noted that (i) at least two pores have been analyzed for each condition and similar results were found in each case, (ii) each pore contained at least $1 \times 10^{6}$ pairs of principal curvatures and (iii) the typical relative error for the estimation of $\left\langle\kappa_{1}\right\rangle_{\text {tot }}$ and $\left\langle\kappa_{1}\right\rangle_{\geqslant 0}$ is about $10 \%$ but is substantially larger (typically $50 \%$ ) for

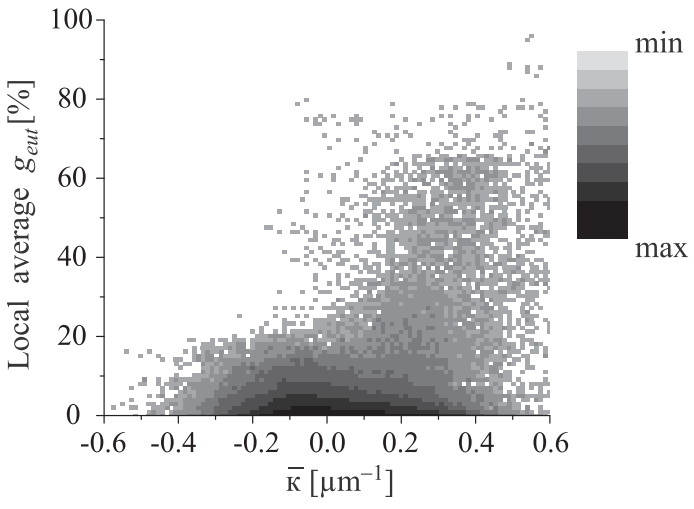

Fig. 6. Probability, indicated with various grey levels, of having a point at the surface of a pore with a given local mean curvature and eutectic fraction. Al- 4.5 wt. $\% \mathrm{Cu}$ alloy, solidified with the $D S_{1 \mathrm{D}-\mathrm{ATM}}$ set-up, noninoculated.

$\left\langle\kappa_{2}\right\rangle_{\text {tot }}$ and $\left\langle\kappa_{2}\right\rangle_{\geqslant 0}$. Highly curved regions are less accurately estimated when the radius of curvature reaches the voxel size, which substantially increases the relative error.

\subsection{Relationship between local mean curvature and adjacent material}

In most $\mathrm{Al}-\mathrm{Cu}$ alloys investigated here, the last liquid to solidify has the eutectic composition. Because of the Laplace-Young equation, the local pore mean curvature should be positive at each point where the pore is in contact with the last liquid to solidify, i.e. with the eutectic after solidification. To verify this concept, the local grey value (i.e. composition) of the region next to the pore surface has been put into relation with the local mean curvature of the pore. The average local grey value in a small sphere of 5 voxels radius surrounding each point of the pore surface has been determined. Knowing the corresponding light-grey level of the eutectic region (see Fig. 4), the fraction of eutectic could be estimated and put into relation to the local mean curvature of the pore at this point.

\section{Results and discussion}

First, the local mean curvature $\bar{\kappa}$ of a pore in an $\mathrm{Al}-$ $4.5 \mathrm{wt} . \% \mathrm{Cu}$ alloy has been put into relation to its surrounding local grey value, as described in Section 4.3 (see Fig. 6). The alloy, non-inoculated, was solidified with the $D S_{1 \mathrm{D}-\mathrm{ATM}}$ set-up at a local cooling rate of $10 \mathrm{~K} \mathrm{~s}^{-1}$. The final fraction of eutectic was about $9 \%$. This 2-D diagram shows in a $\left(\bar{\kappa}, g_{\text {eut }}\right)$ space the probability of finding (on the surface of a pore) a given local mean curvature $\bar{\kappa}$ in contact with a given eutectic fraction present in the near-adjacent material. The grey scale is shown on the left. It can be seen that negative values of $\bar{\kappa}$ have a high probability of being in contact with the Al primary phase, whereas positive values of $\bar{\kappa}$ have an increasing probability of being in contact with the interdendritic eutectic.

Since positively curved regions of a pore are primarily in contact with the eutectic, i.e. the last liquid to solidify, this 


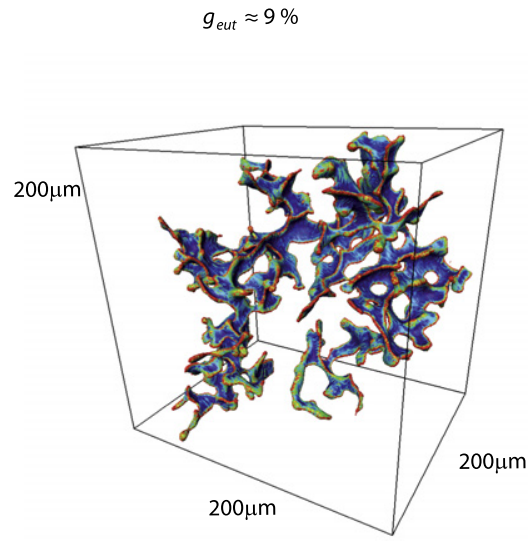

(a)
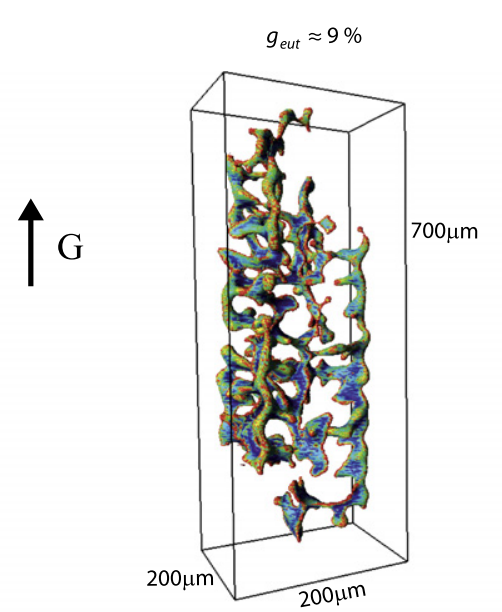

(b)
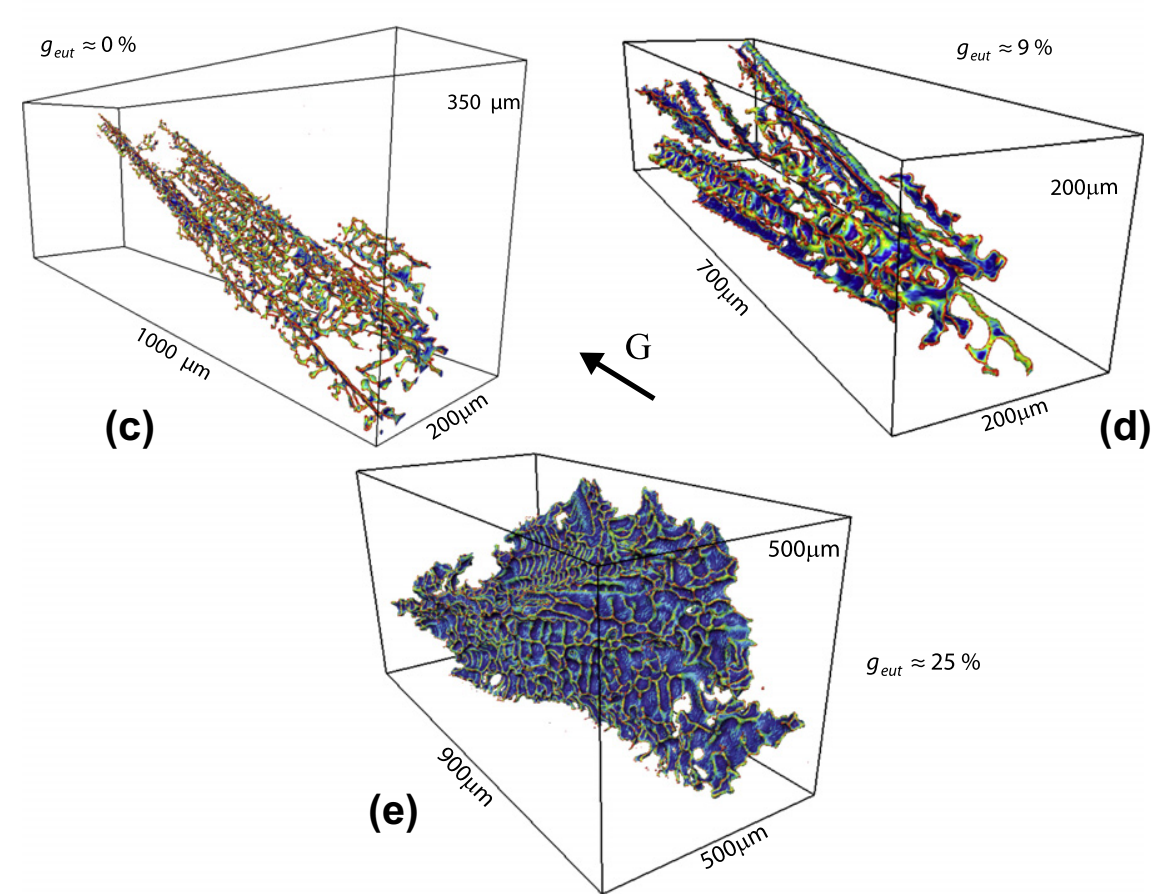

(d)

Fig. 7. 3-D pore morphologies in $\mathrm{Al}-4.5 \mathrm{wt} . \% \mathrm{Cu}$ alloys solidified (a) with and (b) without inoculant in the $D S_{1 \mathrm{D}-\mathrm{ATM}}$ set-up. The cooling rate $\left(\dot{T} \approx-2 \mathrm{~K} \mathrm{~s}^{-1}\right)$, final secondary arm spacing $\left(\lambda_{2} \approx 20 \mu \mathrm{m}\right)$ and eutectic volume fraction $\left(g_{\text {eut }} \approx 9 \%\right)$ are the same in both samples. The figures at the bottom show similar pores formed in $D S_{1 \mathrm{D}-\mathrm{AC}}$ specimens: (c) Al-1 wt. $\% \mathrm{Cu}$ alloy, not inoculated, at $10 \mathrm{~mm}$ from the copper chill, $g_{\text {eut }} \approx 0 \%$ ); (d) Al-4.5 wt. $\% \mathrm{Cu}$ alloy, not inoculated, at $10 \mathrm{~mm}$ from the copper chill, $g_{\text {eut }} \approx 9 \%$ ); (e) Al-10 wt. $\% \mathrm{Cu}$ alloy, not inoculated, at $40 \mathrm{~mm}$ from the copper chill, $g_{\text {eut }} \approx 25 \%$ ). Highly positively curved regions are shown in red, negatively curved regions are blue.

shows that the curvatures measured in post-mortem (i.e. fully solidified) specimens have some relevance to deduce the pinching contribution acting during solidification. Pores formed in a finer structure, with a reduced fraction of eutectic, should have a higher mean curvature for those parts which are in contact with the last liquid, i.e. with the final eutectic. Therefore, post-mortem study of the influence of inoculation conditions, copper content, cooling rate and initial hydrogen content on the final pore morphology offers real potential for estimating the pinching contribution acting during solidification. This is presented in the following.

\subsection{Inoculation influence}

The 3-D shapes of micropores formed in $\mathrm{Al}-4.5 \mathrm{wt} . \% \mathrm{Cu}$ alloys, solidified with and without the addition of inoculant in the $D S_{1 \mathrm{D}-\mathrm{ATM}}$ set-up, are shown in Fig. $7 \mathrm{a}$ and b, respectively. Both specimens were solidified the same day (room temperature of $298 \mathrm{~K}$, relative humidity of $40 \%$ ), with an initial hydrogen composition estimated by Sieverts' law of $0.1 \mathrm{cc}_{\text {STP }} 100 \mathrm{~g}^{-1}$.

As shown in Fig. 7, the pores adopt a very complex shape dictated by the primary phase morphology: it has an "equiaxed" shape in the case of the inoculated alloy 
(Fig. 7a), whereas it is elongated or "columnar" along the (vertical) thermal gradient direction for the non-inoculated alloy (Fig. 7b). The characteristic length scale of the microstructure (i.e. $\lambda_{2}$ ) can clearly be seen in both figures, whereas the typical primary spacing of columnar dendrites $\left(\lambda_{1}\right)$ for the non-inoculated alloy is outlined by the columnar pore. Indeed, after nucleation, the pore grows by gradually replacing and pushing away the liquid phase, while the solid grows where the liquid still remains and pinches the pore: it therefore adopts the 3-D "negative" shape of the primary phase skeleton.

The overall morphology of the pores can be best analyzed in terms of their moments of inertia (see Section 4.1). The dimensionless moment of inertia tensor $I^{N D}$ and corresponding eigenvector matrix $E$ for the equiaxed pore shown in Fig. 7 a are:

$I^{N D}=\left(\begin{array}{ccc}0.81 & 0.00 & 0.00 \\ 0.00 & 0.82 & 0.00 \\ 0.00 & 0.00 & 1.37\end{array}\right) \quad E=\left(\begin{array}{ccc}-0.99 & -0.01 & 0.00 \\ -0.01 & 0.99 & 0.00 \\ 0.00 & 0.00 & 1.00\end{array}\right)$

whereas for the columnar pore of the non-inoculated alloy (Fig. 7b), we have:

$I^{N D}=\left(\begin{array}{ccc}1.35 & 0.00 & 0.00 \\ 0.00 & 1.07 & 0.00 \\ 0.00 & 0.00 & 0.59\end{array}\right) \quad E=\left(\begin{array}{ccc}0.49 & -0.86 & -0.07 \\ 0.86 & 0.49 & -0.08 \\ 0.11 & 0.02 & 0.99\end{array}\right)$

For the columnar pore, the lowest moment of inertia is well aligned along $z$, as expected from its shape, whereas, for the equiaxed pore of Fig. 7a, the tensor is already diagonal in the $x y z$-reference frame and has the largest inertia moment along the thermal gradient. The tensor $I^{N D}$ is nearly degenerate in the $x y$-plane, i.e. perpendicular to $G$. This is probably due to the growth mode of equiaxed grains growing in a thermal gradient. The pore is more or less following the grain boundaries in this case, as can be guessed from Fig. 7a.

Let us now focus on the curvature of these pores. While the characteristic length scale of both microstructures shown in Fig. 7a and b is similar, the pore of the inoculated alloy (Fig. 7a) exhibits more negatively curved (blue) regions than the elongated pore of Fig. $7 \mathrm{~b}$. This can be verified in Table 1, where average curvatures calculated with the method described in Section 4.2 are listed. These values can also be compared with the radius of curvature calculated with the model of Couturier et al. [1], with $g_{\text {eut }}=9 \%$ and $\lambda_{2}=20 \mu \mathrm{m}$. Looking at the three first lines of Table 1, it can be seen that $\left\langle\kappa_{1}\right\rangle_{\text {tot }}<0$ and $\left\langle\kappa_{2}\right\rangle_{t o t}>0$ for both alloys. On the other hand, $\left\langle\kappa_{1}\right\rangle_{\text {tot }}^{\text {Inoculated }}<\left\langle\kappa_{1}\right\rangle_{\text {tot }}^{\text {Non Inoculated }}$ and $\left\langle\kappa_{2}\right\rangle_{\text {tot }}^{\text {Inoculated }}<\left\langle\kappa_{2}\right\rangle_{\text {tot }}^{\text {Non Inoculated }}$, thus explaining that the pore in Fig. 7a is more blue than that in Fig. 7b. Therefore, a pore constrained to grow in an inoculated alloy exhibits more negatively curved regions than a pore constrained to grow in a non-inoculated alloy, with otherwise similar thermal conditions and secondary dendrite arm spacing. This can probably be explained by the fact that, for the inoculated equiaxed microstructure, the pore adopts a negative curvature dictated by both the secondary
Table 1

Inoculant influence on the pore morphology in $\mathrm{Al}-4.5 \mathrm{wt} . \% \mathrm{Cu}$ alloys (same pores as in Fig. 7a and b). The last line gives the surface-to-volume ratio $S_{V}$ of the pore and all the data are given in $\mu \mathrm{m}^{-1}$. The typical cooling rate of $-2 \mathrm{~K} \mathrm{~s}^{-1}$ and thermal gradient of about $5000 \mathrm{~K} \mathrm{~m}^{-1}$ lead to a typical $\lambda_{2}$ spacing of about $20 \mu \mathrm{m}$. Note finally that $g_{\text {eut }} \approx 9 \%$ and that $C_{H 0}=0.1 \mathrm{cc}_{\mathrm{STP}} 100 \mathrm{~g}^{-1}$ in both cases.

\begin{tabular}{lcc}
\hline & Inoculated & Non-inoculated \\
\hline$\left\langle\kappa_{1}\right\rangle_{\text {tot }}$ & -0.104 & -0.080 \\
$\left\langle\kappa_{2}\right\rangle_{t o t}$ & 0.267 & 0.344 \\
$\langle\bar{\kappa}\rangle_{\text {tot }}$ & 0.081 & 0.132 \\
$\left\langle\kappa_{1}\right\rangle_{\geqslant 0}$ & 0.060 & 0.062 \\
$\left\langle\kappa_{2}\right\rangle_{\geqslant 0}$ & 0.574 & 0.546 \\
$\langle\bar{\kappa}\rangle_{\geqslant 0}$ & 0.317 & 0.304 \\
$1 / \bar{R}_{p}$ (using [1]) & 0.333 & 0.333 \\
$S_{V}$ & 0.517 & 0.465 \\
\hline
\end{tabular}

dendrite arms and the overall spherical envelope of the grains.

Looking now at the next three lines of Table 1, we can see that $\langle\bar{\kappa}\rangle_{\geqslant 0}$ is similar for both alloys. Indeed, for otherwise similar conditions, it is expected that the curvature of the pore for regions still in contact with the liquid should only depend on the secondary arm spacing and liquid (or eutectic) volume fraction, and not on the overall shape of the grains. The value of $\langle\bar{\kappa}\rangle_{\geqslant 0}$ is in very good agreement with the value calculated with the model of Couturier et al. [1] (next line of the table).

The overall shape of a pore can be analyzed best if one also looks at the corresponding ISD plots of Fig. 8a and b. The triangle indicates the maximum of the ISD, the open circle the mean value $\left(\left\langle\kappa_{1}\right\rangle_{t o t},\left\langle\kappa_{2}\right\rangle_{t o t}\right)$, and the black square the mean of the positive values $\left(\left\langle\kappa_{1}\right\rangle \geqslant 0,\left\langle\kappa_{2}\right\rangle \geqslant 0\right){ }^{6}$ Finally, the star corresponds to the value calculated with Couturier's model.

In addition to the good agreement between the location of the star with the filled square for both alloys, the distribution for the non-inoculated alloy (Fig. 8b) is mainly elongated along the vertical axis, indicating that a large portion of the pore surface is well represented by cylinders. This branch is also observed for the inoculated alloy on the left, but another smaller branch also extends along the horizontal axis of the ISD plot $\left(\kappa_{2} \approx 0\right.$ and $\left.\kappa_{1} \leqslant 0\right)$. This corresponds to the more "blueish" appearance of the pore in Fig. 7 a.

As a first conclusion, Fig. 8 indicates that the positive curvature of the pores can be fairly well approximated by cylinders, independently of the inoculation condition, and the mean positive curvature is in fairly good agreement with the value obtained from the pinching model of Couturier et al. [1].

\footnotetext{
${ }^{6}$ It is not obvious at first sight that the black square is indeed the mean $\langle\bar{\kappa}\rangle_{\geqslant 0}$. This is due to the grey scale adopted for the representation of the whole ISD plot, which does not show well the positive values only.
} 

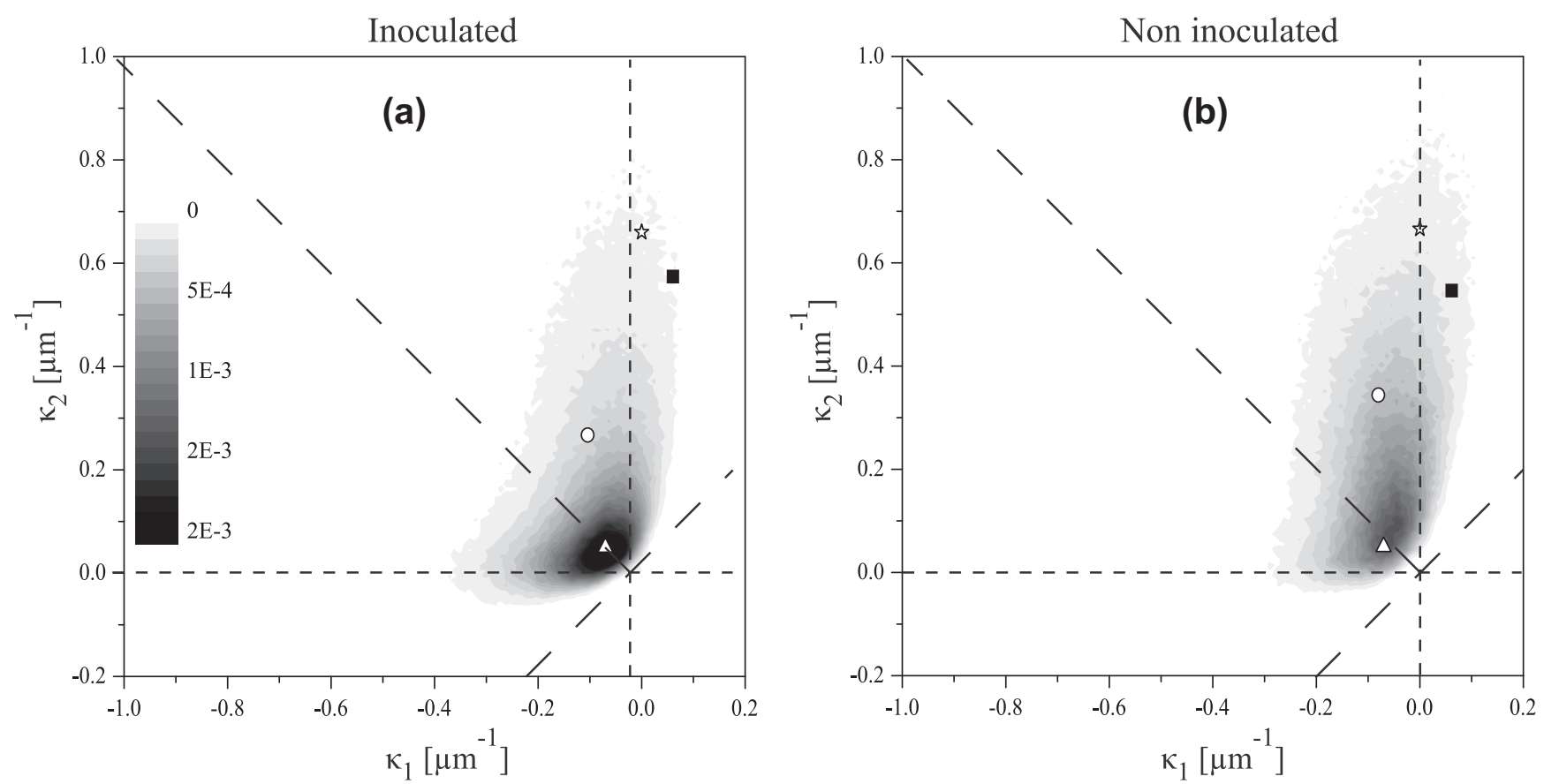

Fig. 8. Interfacial shape distribution (ISD) of pores formed in (a) an inoculated $\mathrm{Al}-4.5 \mathrm{wt} . \% \mathrm{Cu}$ network and (b) a non-inoculated Al-4.5 wt. $\% \mathrm{Cu}$ network (same pores as in Fig. $7 \mathrm{a}$ and $\mathrm{b}$, with $g_{\text {eut }} \approx 9 \%$ in both cases). The white circle represents the mean of the distribution and the triangle its maximum. The black square represents the mean of the distribution if only positive values of both $\kappa_{1}$ and $\kappa_{2}$ are considered. Finally, the star represents the value of the curvature as calculated with the pinching model of Ref. [1].

Table 2

Measured and calculated $\lambda_{2}$ values for the three alloy samples extracted at 10 and $40 \mathrm{~mm}$ from the copper chill, as a function of the alloy copper content. The mean value of the measured cooling rate $|\dot{T}|=\frac{T_{\text {liq }}-T_{\text {eut }}}{t_{\text {liq }}-t_{\text {eut }}}$ is also given for each case.

\begin{tabular}{|c|c|c|c|c|c|c|}
\hline \multirow{3}{*}{$\begin{array}{l}\text { Distance } \\
\text { from } \mathrm{Cu} \\
\text { chill } \\
\text { Cooling } \\
\text { rate }|\dot{T}|\end{array}$} & \multicolumn{2}{|c|}{$\begin{array}{l}\mathrm{Al}-1 \text { wt. } \% \mathrm{Cu} \\
g_{\text {eut }} \approx 0 \%\end{array}$} & \multicolumn{2}{|c|}{$\begin{array}{l}\mathrm{Al}-4.5 \mathrm{wt} . \% \mathrm{Cu} \\
g_{\text {eut }} \approx 9 \%\end{array}$} & \multicolumn{2}{|c|}{$\begin{array}{l}\mathrm{Al}-10 \mathrm{wt} . \% \mathrm{Cu} \\
g_{\text {eut }} \approx 25 \%\end{array}$} \\
\hline & $10 \mathrm{~mm}$ & $40 \mathrm{~mm}$ & $10 \mathrm{~mm}$ & $40 \mathrm{~mm}$ & $10 \mathrm{~mm}$ & $40 \mathrm{~mm}$ \\
\hline & $6 \mathrm{~K} \mathrm{~s}^{-1}$ & $1 \mathrm{~K} \mathrm{~s}^{-1}$ & $7 \mathrm{~K} \mathrm{~s}^{-1}$ & $1.5 \mathrm{~K} \mathrm{~s}^{-1}$ & $10 \mathrm{~K} \mathrm{~s}^{-1}$ & $2 \mathrm{~K} \mathrm{~s}^{-1}$ \\
\hline $\begin{array}{r}\text { Measured } \\
\lambda_{2}(\mu \mathrm{m})\end{array}$ & 26 & 38 & 23 & 32 & 14 & 26 \\
\hline $\begin{array}{c}\text { Calculated } \\
\lambda_{2}(\mu \mathrm{m})\end{array}$ & 31 & 40 & 23 & 32 & 19 & 27 \\
\hline
\end{tabular}

\subsection{Copper content influence}

The curvature of pores growing in between dendrite arms of the primary phase must be influenced by (i) the secondary dendrite arm spacing $\lambda_{2}$ and (ii) the amount of liquid left in between when they form, which, near the end of solidification, is $g_{\text {eut }}$. Therefore, in this section we treat the influence of the copper content of the alloy and of the cooling rate on the pore curvature.

Figs. $7 \mathrm{c}-\mathrm{e}$ show the volume rendering of three pores solidified with the $D S_{1 \mathrm{D}-\mathrm{AC}}$ set-up and colored by their local mean curvature with the same scale. They were obtained in three non-inoculated alloys exhibiting nearly the same secondary arm spacing, but showing different
Table 3

Copper content and cooling rate influence on pore curvatures (data given in $\left(\mu \mathrm{m}^{-1}\right)$ ). The values under the composition are the initial hydrogen compositions estimated with Sieverts' law and volume fraction of eutectic. The last line gives the surface volume ratio of each pore.

\begin{tabular}{|c|c|c|c|c|c|c|}
\hline & \multicolumn{2}{|c|}{$\begin{array}{l}\text { Al-1 wt. } \% \mathrm{Cu} \\
0.51 \mathrm{cc}_{\mathrm{STP}} \\
100 \mathrm{~g}^{-1} \\
g_{\text {eut }} \approx 0 \%\end{array}$} & \multicolumn{2}{|c|}{$\begin{array}{l}\mathrm{Al}-4.5 \mathrm{wt} . \% \mathrm{Cu} \\
0.41 \mathrm{cc}_{\mathrm{STP}} \\
100 \mathrm{~g}^{-1} \\
g_{\text {eut }} \approx 9 \%\end{array}$} & \multicolumn{2}{|c|}{$\begin{array}{l}\mathrm{Al}-10 \mathrm{wt} . \% \mathrm{Cu} \\
0.30 \mathrm{cc}_{\mathrm{STP}} \\
100 \mathrm{~g}^{-1} \\
g_{\text {eut }} \approx 25 \% \\
\end{array}$} \\
\hline & $10 \mathrm{~mm}$ & $40 \mathrm{~mm}$ & $10 \mathrm{~mm}$ & $40 \mathrm{~mm}$ & $10 \mathrm{~mm}$ & $40 \mathrm{~mm}$ \\
\hline$|\dot{T}|\left(\mathrm{K} \mathrm{s}^{-1}\right)$ & 6 & 1 & 7 & 1.5 & 10 & 2 \\
\hline$\lambda_{2}(\mu \mathrm{m})$ & 26 & 38 & 23 & 32 & 14 & 26 \\
\hline $\begin{array}{l}\left\langle\kappa_{1}\right\rangle_{t o t} \\
\left\langle\kappa_{2}\right\rangle_{t o t} \\
\langle\bar{\kappa}\rangle_{t o t}\end{array}$ & $\begin{array}{l}-0.076 \\
0.444 \\
0.184\end{array}$ & $\begin{array}{l}-0.071 \\
0.242 \\
0.086\end{array}$ & $\begin{array}{l}-0.127 \\
0.389 \\
0.131\end{array}$ & $\begin{array}{l}-0.098 \\
0.253 \\
0.077\end{array}$ & $\begin{array}{l}-0.136 \\
0.403 \\
0.134\end{array}$ & $\begin{array}{l}-0.139 \\
0.170 \\
0.015\end{array}$ \\
\hline $\begin{array}{l}\left\langle\kappa_{1}\right\rangle \geqslant 0 \\
\left\langle\kappa_{2}\right\rangle \geqslant 0 \\
\langle\bar{\kappa}\rangle \geqslant 0 \\
\left.1 / \bar{R}_{p} \text { (via }[1]\right)\end{array}$ & $\begin{array}{l}0.066 \\
0.629 \\
0.348 \\
1.538\end{array}$ & $\begin{array}{l}0.047 \\
0.415 \\
0.231 \\
1.053\end{array}$ & $\begin{array}{l}0.064 \\
0.654 \\
0.359 \\
0.289\end{array}$ & $\begin{array}{l}0.051 \\
0.483 \\
0.267 \\
0.208\end{array}$ & $\begin{array}{l}0.068 \\
0.674 \\
0.371 \\
0.325\end{array}$ & $\begin{array}{l}0.051 \\
0.503 \\
0.277 \\
0.175\end{array}$ \\
\hline$S_{V}$ & 0.790 & 0.444 & 0.862 & 0.488 & 0.605 & 0.177 \\
\hline
\end{tabular}

final fraction of eutectic (see Table 2). In Fig. 7d, for example, the $\mathrm{Al}-4.5 \mathrm{wt} . \% \mathrm{Cu}$ sample taken at $10 \mathrm{~mm}$ from the copper chill has a large solidification interval but the cooling rate is higher, whereas in Fig. $7 \mathrm{e}$, the $\mathrm{Al}-10 \mathrm{wt} . \% \mathrm{Cu}$ sample taken at $40 \mathrm{~mm}$ from the copper chill has a shorter solidification interval but a reduced cooling rate. In the first case, $g_{\text {eut }}=9 \%$ and $\lambda_{2}=23 \mu \mathrm{m}$, whereas in the second case, $g_{\text {eut }}=25 \%$ and $\lambda_{2}=26 \mu \mathrm{m}$. The secondary arm spacings, measured directly from the X-ray microtomography, are 

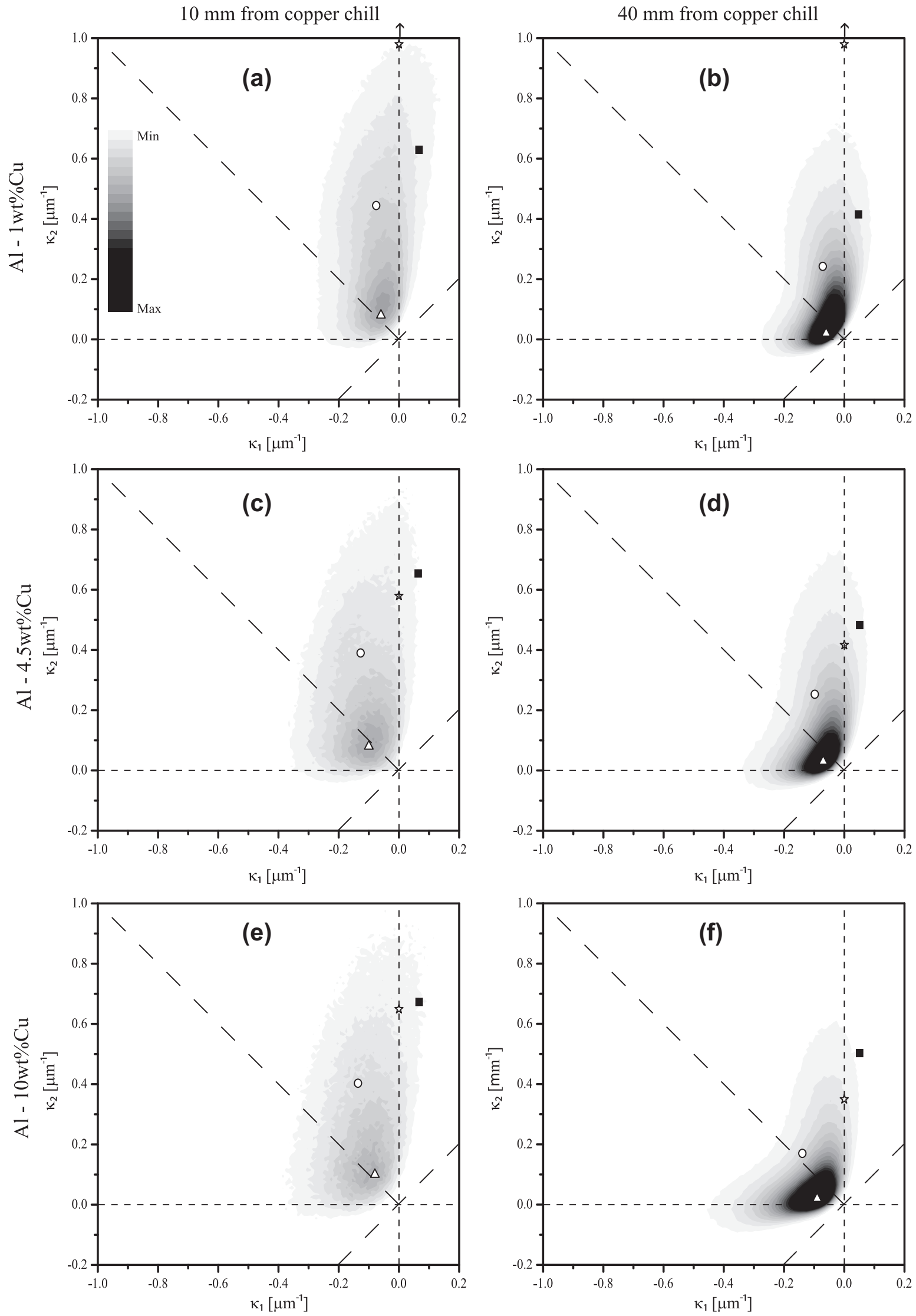

Fig. 9. Influence of cooling rate (or $\lambda_{2}$ ) and copper content ( or $g_{\text {eut }}$ ) on the ISD plots of the pores measured in the three alloys, at two positions from the chill. The symbols are identical to those of Fig. 8. 
in good agrement with those calculated with the coarsening law using a simple 1-D microsegregation model [33] and the measured values of the thermal gradient and liquidus velocity (see Fig. 3). The fractions of eutectic have also been reported in Fig. 2.

Looking at Fig. 7c-e, the pore looks increasingly "red" in these figures. Since the $\lambda_{2}$ spacings are similar, this shows that the pore curvature also depends on the fraction of primary phase.

For the three alloys and two specimens each taken at two locations of the corresponding $D S_{1 \mathrm{D}-\mathrm{AC}}$ ingot, Table 3 summarizes the influence of copper content and cooling rate on the pore curvature values. The initial hydrogen content (estimated by Sieverts' law with the partial pressure of hydrogen in the set-up and the initial melt temperature) is also mentioned in the table. Although the holding temperature of the melt and the hydrogen partial pressure in the surrounding atmosphere were the same for all three specimens, the estimated hydrogen content in the melt (second line in Table 3) in each alloy decreases as the copper content increases. This is due to the influence of solute elements on the solubility limit of hydrogen in aluminum alloys [19].

The conclusions that can be drawn from Table 3 are:

(i) $\langle\bar{\kappa}\rangle_{\text {tot }}$ and $\langle\bar{\kappa}\rangle_{\geqslant 0}$ increase with a decreasing $\lambda_{2}$ for a given copper content, i.e. nearly equal fraction of primary phase,

(ii) $\left\langle\kappa_{1}\right\rangle_{\geqslant 0} \approx 0$ and $\left\langle\kappa_{2}\right\rangle \geqslant 0 \gg 0$ in each case, leading to a possible approximation of the pore curvatures by that of cylinders,

(iii) For identical $\lambda_{2}$ spacings, e.g. Al-1 wt. $\% \mathrm{Cu}$ at $10 \mathrm{~mm}$ and $\mathrm{Al}-10 \mathrm{wt} . \% \mathrm{Cu}$ at $40 \mathrm{~mm}\left(\lambda_{2}=26 \mu \mathrm{m}\right)$, the mean positive curvature $\langle\bar{\kappa}\rangle_{\geqslant 0}$ decreases as the copper content increases, as already observed in Fig. 7c and e.

The trends observed in Table 3 can be seen in the corresponding ISD plots shown in Fig. 9. The same symbols adopted in Fig. 8 for the maximum (triangle), the mean value (open circle), the mean of the positive curvatures (filled square) and the value calculated with Couturier's model (star) have been used again here. As can be seen, all six ISD plots exhibit a main "tail" along the $\kappa_{1}=0$ axis, thus meaning again that the pores can be fairly well approximated by cylinders growing in between dendrite arms. Furthermore, the analytical value calculated with Couturier's model (star) approximates fairly well the mean curvature of the positively curved parts of the pore (filled square), except for the very low composition alloy. However, in this alloy, the volume fraction of eutectic is very small and thus the pore must form before the end of solidification, at some unknown fraction of primary phase.

Finally, the influence of the initial hydrogen content on the curvatures of pores contained in $D S_{1 \mathrm{D}-\mathrm{AC}}$ castings solidified under the same conditions has been investigated [21]. However, no significant influence, besides the amount and overall size of the pores, was found.

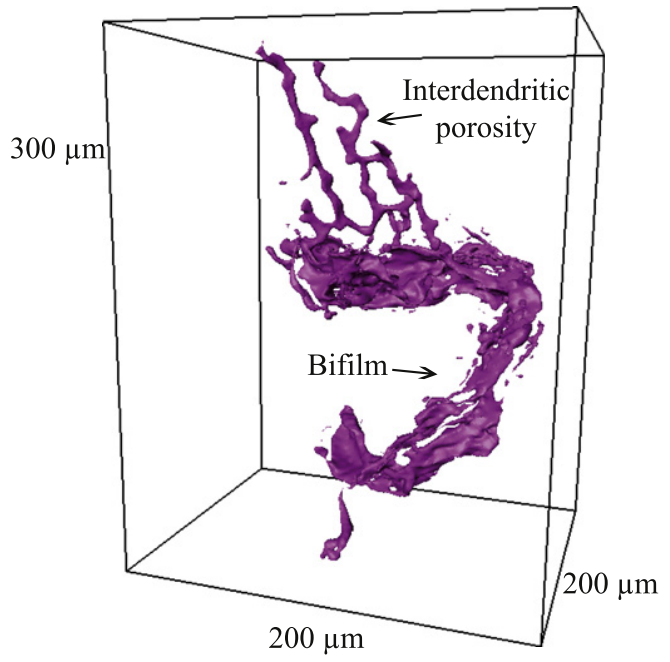

Fig. 10. Interdendritic microporosity originating from an oxide bifilm in Al-4.5 wt. $\% \mathrm{Cu}$ alloy, cast with the $D S_{1 \mathrm{D}-\mathrm{AC}}$ set-up

\subsection{Bifilms}

A final remark is now made on the influence of oxide bifilms. The main difference between the two DS set-ups is that melt pouring was not required with the $D S_{1 \mathrm{D}-\mathrm{ATM}}$ set-up, while it was for the $D S_{1 \mathrm{D}-\mathrm{AC}}$ set-up. A comparison of the pores of Fig. $7 b$ and d, obtained for the same alloy but cast with the two different set-ups, does not show a significant difference: both pores are elongated along the thermal gradient, since the alloys were non-inoculated, similar moment of inertia tensors were found, and no evidence of bifilm could be seen.

However, as shown in Fig. 10, we have also observed "unconventional" pore morphologies (in very few cases, less than 5\%), especially when the initial hydrogen content was low. This particular pore morphology is most probably due to an entrapped oxide skin or oxide bifilm from which an interdendritic pore has grown. Although this assumption should be assessed by in situ solidification experiments, it is the most plausible explanation for such a peculiar pore shape, since the latter was only observed in samples cast with the $D S_{1 \mathrm{D}-\mathrm{AC}}$ set-up, i.e. when melt pouring was required. To the authors' knowledge, this is the first time that the 3-D volume rendering of such an interdendritic pore, originating from an entrapped oxide bifilm, has been reported with such a high resolution $(0.74 \mu \mathrm{m}$ voxel $\left.^{-1}\right)$. Note the small dimensions of the bifilm and the even finer shape of the interdendritic microporosity originating from it, compared to the "regular" pores observed in most other cases. This result confirms the mechanism that pores can nucleate on oxide bifilms, as proposed by Campbell [2], but this also shows that this mechanism is definitely not universal. Pores certainly nucleate heterogeneously, on some impurities or even oxide particles, but not necessarily bifilms (unless some bifilms are smaller than the resolution of our X-ray tomography measurements, i.e. micron). 


\section{Conclusions}

In summary, the following conclusions can be drawn from this study:

(i) The pore morphology adopts the 3-D negative shape of the primary solid. In inoculated alloys, the pores are equiaxed, whereas in non-inoculated alloys with a columnar structure, the pores are columnar. They are elongated along the primary trunk direction of the dendrites, which approximately corresponds to the thermal gradient.

(ii) The curvature distribution of micropores in noninoculated and inoculated alloys exhibit a main branch along the $\kappa_{1}=0, \kappa_{2}>0$ axis. This shows that the fine structure of the pores can be fairly well approximated by a set of cylinders growing in between the dendrite arms.

(iii) Pinching of pores by the solid phase was shown to be primarily dependent on the volume fraction of the primary phase $g_{\alpha}$ and on the secondary dendrite arm spacing $\lambda_{2}$. While critical for the total amount of porosity and the overall pore size, the initial hydrogen content has a negligible influence on the curvature of the pores.

(iv) The model of a non-wetting pore pinched by a pack of cylindrical dendrite arms developed by Couturier et al. [1] gives a fairly good and simple approximation to the curvature distribution of actual pores in $\mathrm{Al}-\mathrm{Cu}$ alloys. The non-agreement observed at low copper content (i.e. for $g_{\text {eut }} \ll 1$ ) was attributed to the fact that the pores formed before any eutectic could form. It is clear that in an alloy without any eutectic, some cut-off of the analytical expression of Couturier's model must be introduced as infinite overpressure is to be avoided.

(v) Very high values of the local mean curvature of a pore in contact with the eutectic - assumed to be the last curvature of the pore when it was in contact with the liquid - have been measured. Curvature values as high as $0.35 \mu \mathrm{m}^{-1}$ have been measured, which gives a pore overpressure near the end of solidification $\left(2 \gamma_{\ell g} \bar{\kappa}\right)$ of about $600 \mathrm{kPa}$. This shows the importance of the curvature term in any microporosity model. It also means that the interdendritic liquid can have fairly large negative pressure, while the pressure in the pores is of course positive. This is consistent with the porosity calculations of Couturier et al. [17].

\section{Acknowledgments}

The X-ray tomography experiments have been conducted at the ESRF in Grenoble (France) under the project
ANR-05-BLAN-0286-01 TOMOSOLIDAL supported by the 'Agence Nationale de la Recherche', and at the SLS in Villigen (Switzerland) under the project ID20090118. The authors thank E. Boller (ESRF), A. Phillion (LSMX), L. Salvo and M. Suéry (INP-Grenoble), M. Stampanoni and S. McDonald (SLS) for assistance with X-ray tomography experiments. The authors also thank J.-D. Wagnière (LSMX) for the development of the induction furnace.

\section{References}

[1] Couturier G, Rappaz M. In: Light metals. San Antonio (TX): TMS 2006. p. 143.

[2] Campbell J. Castings. 3rd ed. Oxford: Butterworth Heinemann; 2003.

[3] Dantzig JA, Rappaz M. Solidification. Lausanne: EPFL Press; 2009.

[4] Lee PD, Chirazi A, See D. J Light Met 2001;1:15.

[5] Carlson KD, Lin Z, Beckermann Ch. Metall Mater Trans B 2007;38:541.

[6] Felberbaum M, Landry-Désy E, Weber L, Rappaz M. Acta Mater 2011;59:2302.

[7] Carpentier D. PhD thesis. INPL Nancy; 1994.

[8] Poirier DR, Yeum K, Maples AL. Metall Trans A 1987;18:1979.

[9] Péquet Ch, Gremaud M, Rappaz M. Metall Mater Trans A 2002;33:2095.

[10] Baruchel J, Buffière J-Y, Cloetens P, Di Michiel M, Ferrie E, Ludwig W, et al. Scripta Mater 2006;55:41.

[11] Mathiesen RH, Arnberg L, Ramsøskar K, Weitkamp T, Rau C, Snigirev A. Metall Mater Trans B 2002;33:613.

[12] Wang JS, Lee PD. Int J Cast Metal Res 2007;20:151.

[13] Lee PD, Hunt JD. Acta Mater 1997;45:4155.

[14] Limodin N, Salvo L, Boller E, Suéry M, Felberbaum M, Gailliègue S, et al. Acta Mater 2009;57:2300.

[15] Terzi S, Salvo L, Suéry M, Limodin N, Adrien J, Maire E, et al Scripta Mater 2009;61:449.

[16] Piwonka TS, Flemings MC. Trans AIME 1966;236:1157.

[17] Couturier G, Rappaz M. Model Simul Mater Sci Eng 2006;14:253.

[18] Anyalebechi PN. In: Light metals. Warrendale (PA): TMS; 2003. p. 857.

[19] Sigworth GK, Engh TA. Metall Mater Trans B 1982;13:447.

[20] Kammer D, Voorhees PW. Acta Mater 2006;54:1549.

[21] Felberbaum M. PhD thesis. EFPL Lausanne; 2010.

[22] Zhao HS, Wu CZ, Li YY. Int J Cast Metal Res 2008;21:313.

[23] Apprill JM, Poirier DR, Maguire MC, Gutsch TC. Mater Res Soc Proc 1998;521:291.

[24] Fredriksson H, Svensson I. Metall Mater Trans B 1976;7:599.

[25] Drezet J-M, Rappaz M, Grün GU, Gremaud M. Metall Mater Trans A 2000;31:1627.

[26] Salvo L, Cloetens P, Maire E, Zabler S, Blandin JJ, Buffière J-Y, et al Nucl Instrum Method Phys Res B 2003;200:273.

[27] Maire E, Buffière J-Y, Salvo L, Blandin JJ, Ludwig W, Letang JM. Adv Eng Mater 2001;3:539.

[28] Kak AC, Slaney M. Principles of computerized tomography imaging. Philadelphia (PA): IEEE Press; 1987.

[29] http://rsbweb.nih.gov/ij/index.html.

[30] Doube M, Conroy AW, Christiansen P, Hutchinson JR, Shefelbine S. PLoS ONE 2009;4:4742.

[31] Andrew Pressley. Elementary differential geometry. London: Springer-Verlag; 2001.

[33] Clyne TW, Kurz W. Metall Mater Trans A 1981;12:965. 